\title{
No Zero Divisor for Wick Product in $(S)^{*}$
}

\author{
Takahiro HASEBE, Izumi OJIMA and Hayato SAIGO \\ RIMS, Kyoto University, Kyoto 606-8502, Japan
}

\begin{abstract}
In White Noise Analysis (WNA), various random quantities are analyzed as elements of $(S)^{*}$, the space of Hida distributions ([1]). Hida distributions are generalized functions of white noise, which is to be naturally viewed as the derivative of the Brownian motion. On $(S)^{*}$, the Wick product is defined in terms of the $\mathcal{S}$-transform. We have found such a remarkable property that the Wick product has no zero divisors among Hida distributions. This result is a WNA version of Titchmarsh's theorem and is expected to play fundamental roles in developing the "operational calculus" in WNA along the line of Mikusiński's version for solving differential equations.
\end{abstract}

2000 AMS Mathematics Subject Classification: 60H40, 44A40

\section{Introduction}

In the present notes, we verify one of the most essential algebraic properties of the so-called U-functionals consisting of the $\mathcal{S}$-transforms of the Hida distributions in the context of white noise analysis (WNA, for short): they constitute an integral domain, that is, a ring with no zero divisors. For this purpose, we start from the Gel'fand triple,

$$
S(\mathbb{R}) \subset L^{2}(\mathbb{R}) \subset S^{\prime}(\mathbb{R}),
$$

together with the Gauss measure $\mu$ on $S^{\prime}(\mathbb{R})$ determined through the BochnerMinlos theorem by its characteristic function:

$$
\int_{S^{\prime}(\mathbb{R})} e^{i\langle x, f\rangle} d \mu(x)=e^{-\frac{1}{2}\langle f, f\rangle} .
$$

As usual, $S(\mathbb{R})$ and $S^{\prime}(\mathbb{R})$ here denote the spaces of rapidly decreasing Schwartz test functions and of Schwartz tempered distributions, respectively. $\left(S^{\prime}(\mathbb{R}), \mu\right)$ is the space of white noise.

Extending test functions from $S(\mathbb{R})$ to $L^{2}(\mathbb{R})$, we obtain Wiener's Brownian motion in such a form as

$$
B_{t}:=\left\langle x, 1_{[0, t]}\right\rangle
$$


Then a "higher Gel'fand triple" can be constructed as follows [1]:

$$
(S) \subset\left(L^{2}\right):=L^{2}\left(S^{\prime}(\mathbb{R}), \mu\right) \subset(S)^{*},
$$

where the elements of $(S)$ and $(S)^{*}$ are called, respectively, "white noise test functionals" and "generalized white noise functionals", the latter of which are also called "Hida distributions".

\section{Definitions of $\mathcal{S}$ - and $\mathcal{T}$-transforms}

In constructing the framework of calculus on these spaces, the $\mathcal{S}$-transform [2] defined by

$$
\mathcal{S}:\left(L^{2}\right) \ni \phi \mapsto(\mathcal{S} \phi)(\xi):=\int_{S^{\prime}(\mathbb{R})} \phi(x+\xi) d \mu(x) \quad \text { for } \xi \in S(\mathbb{R}),
$$

plays crucial roles, especially in our context aiming at an infinite-dimensional version of "operational calculus". The domain $\left(L^{2}\right)$ of $\mathcal{S}$ can be extended to $(S)^{*}$. Similarly, the $\mathcal{T}$-transform is introduced by

$$
\mathcal{T}:\left(L^{2}\right) \ni \phi \mapsto(\mathcal{T} \phi)(\xi):=\int_{S^{\prime}(\mathbb{R})} \phi(x) e^{i\langle x, \xi\rangle} d \mu(x) \quad \text { for } \xi \in S(\mathbb{R}),
$$

and its domain $\left(L^{2}\right)$ is also extended to $(S)^{*}$. Both $\mathcal{S}$ and $\mathcal{T}$ are injective.

\section{CCR structure of Hida derivatives and a new version of quantum decomposition}

We can define Hida derivative $\partial_{t}:(S) \longrightarrow(S)$ by

$$
\partial_{t}: \phi(x) \mapsto \mathcal{S}^{-1}\left\{\frac{\delta}{\delta \xi(t)}(\mathcal{S} \phi)(\xi)\right\}(x),
$$

which satisfies the following equality for all $\phi \in(S)$ :

$$
\partial_{t} \phi(x)=D_{\delta_{t}} \phi(x):=\lim _{\varepsilon \rightarrow 0} \frac{\phi\left(x+\varepsilon \delta_{t}\right)-\phi(x)}{\varepsilon} .
$$

The adjoint $\partial_{t}^{*}:(S)^{*} \longrightarrow(S)^{*}$ of $\partial_{t}$ can be identified with the map:

$$
\partial_{t}^{*}: \phi(x) \longmapsto \mathcal{S}^{-1}\{\xi(t)(\mathcal{S} \phi)(\xi)\}(x) .
$$

With the notation $x_{t}:=\partial_{t}^{*} 1 \in(S)^{*}$ for the image of the identity functional $1 \in(S)^{*}$ under $\partial_{t}^{*}$, we have the relation $x(t)=\frac{d}{d t} B(t)$ valid for the $C^{\infty}$ mappings $x(t): t \mapsto x_{t}$ and $B(t): t \mapsto B_{t}$, and hence, $x_{t}$ is called "the white noise at $t "$. 
It is important that $\partial_{t}$ and $\partial_{t}^{*}$ satisfy the canonical commutation relations. Moreover, they satisfy the relation

$$
x_{t} \cdot=\partial_{t}+\partial_{t}^{*}
$$

as operators $(S) \longrightarrow(S)^{*}$. Here an operator $x_{t}$. on $(S)$ to multiply the random variable $x_{t}$ is defined by

$$
\left\langle\left\langle x_{t} \cdot y, \phi\right\rangle\right\rangle=\left\langle\left\langle x_{t}, y \cdot \phi\right\rangle\right\rangle
$$

in terms of the pointwise product $y \cdot \phi$ of $y$ and $\phi$ and of the duality coupling $\langle\langle\cdot, \cdot\rangle\rangle$ between $(S)^{*}$ and $(S)$. In reference to the "finite-dimensional analog of $\mathcal{S}$-transform" given with $d \mu_{1}(x):=e^{-\frac{x^{2}}{2}} d x / \sqrt{2 \pi}$ by

$$
\mathcal{S}_{1}: \phi(x) \mapsto\left(\mathcal{S}_{1} \phi\right)(\xi):=\int_{\mathbb{R}} \phi(x+\xi) d \mu_{1}(x),
$$

it can be understood that the above definitions and properties are quite natural: under $\mathcal{S}_{1}$, the differential operator $\frac{d}{d x}$ is transformed into $\frac{d}{d \xi}$ and $x-\frac{d}{d x}$ into $\xi$, respectively. Note here that $x-\frac{d}{d x}$ is the adjoint of $\frac{d}{d x}$ with respect to the normal Gauss measure $\mu_{1}$ owing to the relation:

$$
\int_{\mathbb{R}}\left\{\left(x-\frac{d}{d x}\right) f\right\} g e^{-\frac{x^{2}}{2}} d x=\int_{\mathbb{R}} f\left\{\frac{d}{d x} g\right\} e^{-\frac{x^{2}}{2}} d x .
$$

Namely, the differential operators and their adjoints with respect to a reference measure given by the Gaussian one, are not commutative but satisfy canonical commutation relations. In sharp contrast, $\frac{d}{d x}$ and its adjoint $-\frac{d}{d x}$ with respect to the Lebesgue measure are mutually commutative. Moreover, $x-\frac{d}{d x}$ can also be viewed as "a renormalized form of $x$ " due to the non-Haar nature of the Gaussian measure. For instance, $\left(x-\frac{d}{d x}\right)^{n} 1$ is nothing but the $n$-th Hermite polynomial, which can be seen as "the Gaussian version of $x^{n}$ ". In this way, the $\mathcal{S}$-transform naturally extends the algebraic structure found in finite dimensional situations to the infinite dimensional ones.

\section{Absence of zero-divisors}

Using the $\mathcal{S}$-transform again, we can even introduce "the product of Hida distributions". The theorem below plays a key role.

Theorem 1 (Potthoff and Streit [3]) A complex-valued functional $F$ on $S_{c}(\mathbb{R})$ is the $\mathcal{S}-(\mathcal{T}$-)transform of a Hida distribution if and only if it satisfies the following conditions: 
(i) for any $\xi, \eta \in S_{c}(\mathbb{R})$, the function $F(z \xi+\eta)$ is an entire function of $z \in \mathbb{C}$,

(ii) there exist constants $K, a, p \geq 0$ such that

$$
|F(z \xi)| \leq K e^{a|z|^{2}\left\|H^{p} \xi\right\|_{2}^{2}} .
$$

(Here $H$ denotes the Hamiltonian of a harmonic oscillator with the spectrum $\sigma=\{2 k+2 \mid k=0,1,2,3, \cdots\}$.)

Functionals satisfying the above conditions (i),(ii) are called U-functionals. It can be proved that U-functionals form an algebra under the pointwise product. As $\mathcal{S}$ - $(\mathcal{T}$-)transforms are injective, we can define (two types of) products on $(S)^{*}$ :

$$
\begin{aligned}
& a \diamond b:=\mathcal{S}^{-1}(\mathcal{S} a \cdot \mathcal{S} b), \\
& a * b:=\mathcal{T}^{-1}(\mathcal{T} a \cdot \mathcal{T} b),
\end{aligned}
$$

where $\diamond$ and $*$ are called, respectively, "Wick product" and "convolution" with 1 and $\delta_{\mathbf{0}}$ (white noise delta-functional) as their units. From the characterization theorem, we can derive the fundamental property shared by these products:

Theorem 2 The products, $\diamond$ and $*$, have no zero divisor among Hida distributions.

Proof. Since $\mathcal{S}$ - and $\mathcal{T}$-transforms are isomorphic, it suffices to prove that there are no zero divisors among U-funtionals under the pointwise product. We start from the equality $U_{1} U_{2}=0$ for two U-functionals $U_{1}$ and $U_{2}$. This means $U_{1}(f) U_{2}(f)=0$ for all $f \in S(\mathbb{R})$, and we can assume that $U_{1}$ is not identically zero, which implies the existence of $f$ such that $U_{1}(f) \neq 0$. By the condition (i) in the above definition of U-functionals, we can take a sufficiently small $\lambda$ for each $g$ so that $U_{1}(f+\lambda g) \neq 0$ is valid, and hence, $U_{2}(f+\lambda g)=0$. Because of the analyticity expressed in the condition (i), this implies the equality $U_{2}(f+\lambda g)=0$ for all $\lambda$. Since $g$ is arbitrary, $U_{2}$ vanishes identically: $U_{2}=0$.

\section{$5 \quad$ Summary and prospect}

We summarize our results into such a basic scheme of the algebraic structures inherent to WNA: the space $(S)^{*}$ of Hida distributions is mapped by the $\mathcal{S}$ and $\mathcal{T}$-transforms isomorphically into the space of U-functionals. In terms of these transforms, $(S)^{*}$ is equipped with two kinds of commutative products, $\diamond$ and $*$, and is acted upon by the CCR algebra consisting of Hida derivatives 
$\partial_{t}, \partial_{t}^{*}$ which provides the basic random variables $x_{t} \in(S)^{*}$ with its quantum decomposition, $x_{t} \cdot=\partial_{t}+\partial_{t}^{*}$. Finally $(S)^{*}$ as well as its image under the $\mathcal{S}$ and $\mathcal{T}$-transforms constitutes an integral domain without zero divisors. We note that the validity of this result heavily relies upon the complex-analytic structure involved in the framework of WNA.

To take advantage of the above picture, we recall here that Titchmarsh's theorem guarantees the absence of zero divisors with respect to the convolution product among such functions as continuous on $[0, \infty]$ and as vanishing on $(-\infty, 0)$. Heaviside's operational calculus has been formulated by Mikusiński [4] in the use of the usual convolution product. Since the above proposition guarantees the validity of the similar result, we can naturally expect that it will allow us to develop a kind of infinite-dimensional version of "operational calculus" in the context of WNA. As a generalization of the Itô integral, the Hitsuda-Skorohod integral can be introduced in terms of $\partial_{t}^{*} f=x_{t} \diamond f$. Since $(S)^{*}$ has no zero divisors, we can introduce the Wick-inverse of Hida distributions by constructing the quotient field of $(S)^{*}$. They should play crucial roles, for instance, in solving stochastic partial differential equations and in analyzing singularities associated with many distribution-theoretical aspects inherent to WNA [5].

The authors would like to express their sincere thanks to Prof. T. Hida for his encouragement and interest in our joint project on the duality aspects in WNA. They are very grateful to Messrs. R. Harada, H. Ando and K. Nishimura for valuable discussions.

\section{References}

[1] The concept of the generalized white noise functionals was first introduced by Prof. Hida in his lecture notes, Hida, T., Analysis of Brownian functionals, Carleton Math. Notes 13 (1975). In the present note, we use the version due to Kubo, I. and Takenaka, S.: Calculus on Gaussian white noise, I-IV. Proc. Japan Acad. 56 (1980) - 58 (1982).

[2] Hida, T., Kuo, H.-H., Potthoff, J. and Streit, L., White Noise, Kluwer (1993).

[3] Potthoff, J. and Streit, L.: A characterization of Hida distributions, J. Funct. Anal. 101, 212-229 (1991).

[4] Mikusiński, J.: Operational Calculus, Pergamon Press (1959).

[5] Hasebe, T., Ojima, I. and Saigo, H.: in preparation. 